\title{
Evaluation of a Bruker timsTOF Pro for Native Mass Spectrometry
}

Erin M. Panczyk ${ }^{1,2}$, Dalton T. Snyder ${ }^{2}$, Fanny C. Liu ${ }^{3}$, Yu-Fu Lin ${ }^{1,2}$, Mark E. Ridgeway ${ }^{4}$, Melvin A. Park', Christian Bleiholder ${ }^{3,5}$, Vicki H. Wysocki ${ }^{1,2}$

1 Department of Chemistry and Biochemistry, The Ohio State University, Columbus, OH 43210, USA

2 Resource for Native MS Guided Structural Biology, The Ohio State University, Columbus, OH 43210, USA

3 Department of Chemistry and Biochemistry, Florida State University, Tallahassee, FL 32306, USA

4 Bruker Daltonics Inc., Billerica, MA 01821, USA

5 Institute of Molecular Biophysics, Florida State University, Tallahassee, FL 32306, USA

Corresponding Author:

Vicki H. Wysocki

Office Address:

279 Biomedical Research Tower

460 W. $12^{\text {th }}$ Avenue

Columbus, $\mathrm{OH} 43210$

Office phone: $614-292-8687$

Email:wysocki.11@osu.edu 


\begin{abstract}
Mass spectrometry-based assays in structural biology studies typically measure either intact or digested proteins. Traditionally, there are different mass spectrometers dedicated for such measurements: those focused on the rapid analysis of small molecules, such as digested peptides, and those designed for high mass intact analysis. The Bruker timsTOF Pro mass spectrometer (ion mobility-quadrupole-time of flight platform, IM-Q-TOF), has become widely utilized for the analysis of small molecules in the fields of proteomics and metabolomics, with ion mobility spectrometry offering an additional stage of ion separation coupled to liquid chromatography. While this instrument has proven capabilities for small molecule analysis, the ability to perform high-quality native mass spectrometry of intact protein complexes remains largely uninvestigated. Here, we evaluate this IM-Q-TOF platform for the analysis of intact proteins and non-covalently bound protein complexes as small as $12 \mathrm{kDa}$ (cytochrome c) and as large as $801 \mathrm{kDa}$ (GroEL), utilizing the full range of ion mobility, MS, and MS/MS (in-source cleanup and collision cell CID) experiments available on this platform. In-source activation and collision cell CID were found to be robust capabilities for both small and large complexes. Nonetheless, the TIMS analyzer was soft enough to preserve protein-ligand interactions between 1,3-benzenedisulfonamide and carbonic anhydrase. TIMS-CID was performed on the protein complexes streptavidin (53 kDa), avidin (68 kDa) and cholera toxin B (CTB, $58 \mathrm{kDa}$ ). Pyruvate kinase and GroEL were beyond the trapping capabilities of the commercial TIMS analyzer. Although quadrupole selection is limited to $\mathrm{m} / \mathrm{z} 3000$ by the manufacturer, ions of significantly higher $\mathrm{m} / \mathrm{z}$ can be transmitted and studied. The present results show that the commercially available Bruker IM-Q-TOF platform can be used for both omics and native mass spectrometry applications; however, modifications to the commercial RF drivers for the TIMS analyzer and quadrupole will be required if protein complexes greater than a few tens of $\mathrm{kDa}$ are of interest.
\end{abstract}

Key words: timsTOF, native MS, protein complexes 


\section{Introduction}

The determination and understanding of protein structures are critical to unraveling key insights into protein function and possibly malfunction. The field of structural biology consists of several complementary techniques in which different approaches to investigate the structure of these biological assemblies are integrated with physical theories and computational models. ${ }^{1}$ Traditional biophysical techniques employed for the determination of protein structure include $x$-ray crystallography, small angle $x$-ray scattering (SAXS) crystallography, cryo-electron microscopy (cryo-EM), and nuclear magnetic resonance (NMR) spectroscopy. ${ }^{1}$ Additionally, native mass spectrometry (nMS) is an analytical technique increasingly being utilized to answer structural biology questions. ${ }^{2-4}$ Mass spectrometry measurements of intact macromolecular complexes has been made possible due to advancements in sample preparation and instrumentation, including the implementation of electrospray ionization (ESI), in which proteins and protein complexes can be ionized and gently transferred into the gas phase while maintaining solutionlike structures and non-covalent interactions. ${ }^{5-8}$

Early generation mass spectrometers were developed and applied for the analysis of small molecules and required several instrument modifications to expand their capabilities to high mass proteins and protein complexes. ${ }^{9-12}$ The field of nMS, however, has proven to be a valuable analytical technique and mass spectrometry vendors are more commonly developing and commercializing various platforms for large biomolecule analysis. ${ }^{12-13}$ Modifications to mass spectrometers for $\mathrm{nMS}$ applications include the implementation of nanoelectrospray ionization (nanoESI), including robust ion sources for desolvation and desalting of biological samples, low-frequency (high $m / z$ ) RF drivers to extend the mass range of devices, and mass analyzers capable of measuring and resolving high mass species. These modifications, originally performed primarily by individual research groups, have become more prevalent with instrument manufacturers, expanding the field of nMS to a larger number of research groups and industry partners.

In addition to mass measurements and structural information gained from tandem MS experiments, ion mobility spectrometry (IMS) has proven to be an informative analytical tool to probe the gas-phase structures of biological molecules. ${ }^{14-17}$ IMS is a gas-phase separation technique in which ions are separated based on their mobility (a function of an ion's mass, charge, and shape) in a weak electric field and background gas. For Trapped Ion Mobility Spectrometry (TIMS), an electric field that opposes the ion motion is used to hold ions stationary against a bath gas which pushes ions along the TIMS analyzer (an ion funnel). Ions of different mobilities are trapped at different points (potentials) along the ion optical axis using the electric field gradient and are then eluted from the device over time, based on their mobility, as the TIMS potential gradient is reduced. ${ }^{18-23}$ TIMS, or an alternative IM approach called FAIMS, are often coupled with liquid chromatography in "omics" based mass spectrometry experiments, such as proteomics and metabolomics, in which complex biological samples require orthogonal modes of separation to separate and identify the numerous, structurally similar components. ${ }^{18-20,24-25}$ In addition to TIMS serving as a separation technique, the collision cross section (CCS), a rotationally averaged measurement of an ion's structure, can be determined from the measured mobility value, thus providing insight into protein shape and conformation. ${ }^{26,27}$ As a result, mass spectrometers with IMS capabilities are highly desirable for various biological applications.

Here, we evaluate an IM-Q-TOF MS platform initially marketed for small molecule analysis as a platform of nMS studies. The availability of commercial mass spectrometers that can span multiple applications, including both -omics and native experiments, will allow mass spectrometrists to perform more expansive and numerous experiments (such as peptide mapping to identify site specific post translational 
modifications, lipidomics to probe specific ligand binding to endogenous proteins, or CCS measurements of the intact protein) to answer structural biology questions, therefore enhancing efficiency while also being cost-effective. ${ }^{27-29}$

\section{Experimental Section}

\section{Materials}

Ammonium acetate, cytochrome $c$, triethylammonium acetate (TEAA), cholera toxin $B$ (CTB), carbonic anhydrase from bovine erythrocytes (CA), and 1,3-benzenedisulfonamide (BS) were purchased from Sigma Aldrich (St. Louis, MO). GroEL lyophilized powder was also purchased from Sigma and prepared via a refolding procedure as described elsewhere. ${ }^{30}$ Avidin from hen egg-white and streptavidin from Streptomyces avidinii were purchased from Thermo Scientific Pierce Biotechnology (Rockford, IL). Pyruvate kinase from rabbit was purchased from Lee Biosolutions (Maryland Heights, MO).

Protein samples were buffer-exchanged into $200 \mathrm{mM}$ ammonium acetate ( $\mathrm{pH}$ near neutral) with size exclusion chromatography spin columns with a $6 \mathrm{kDa}$ cutoff (Micro Bio-Spin 6, Bio-Rad, Hercules, CA) and were further diluted with $200 \mathrm{mM}$ ammonium acetate to 5-10 $\mu \mathrm{M}$ protein complex concentration. For experiments performed under charge reducing conditions, TEAA was added to protein solutions at a final concentration of $40 \mathrm{mM}$ TEAA and $160 \mathrm{mM}$ ammonium acetate. Cytochrome $\mathrm{c}$ was electrosprayed from a $30 \mu \mathrm{M}$ solution in $10 \mathrm{mM}$ aqueous ammonium acetate.

\section{Instrumentation}

All experiments were performed on a commercial Bruker IM-Q-TOF (timsTOF Pro) mass spectrometer without any hardware modifications. Cytochrome c was directly infused at a flow rate of $180 \mu \mathrm{L} / \mathrm{h}$ using an Apollo II ion source whereas the other proteins and protein complexes were directly infused using nanoelectrospray ( $\mathrm{nESI}$ ) ionization with a Bruker $\mathrm{nESI}$ source. This $\mathrm{nESI}$ source design is identical to that used for native MS experiments on a Bruker SolariX 15 T FT-ICR mass spectrometer. ${ }^{31-34}$ Briefly, the source consists of a linear positioning station in which a glass capillary containing the protein solution was brought into contact with a grounded platinum wire while a DC voltage is applied to the counter entrance electrode of the mass spectrometer. Borosilicate glass capillaries (with filament, Sutter Instruments) were prepared in-house using a Sutter Instruments P-97 pipette puller (Novato, CA) and protein samples were ionized using spray voltages of 0.5-1.0 kV.

To transmit protein complexes, the RF amplitudes for ion transfer elements were increased relative to typical settings for small molecules, while also preventing accidental ion activation. The Transfer Funnel 1 RF was set to $300 \mathrm{~V}_{\mathrm{pp}}$, Transfer Funnel $2 \mathrm{RF}$ was set to $600 \mathrm{~V}_{\mathrm{pp}}$, and the Transfer Multipole RF was set to $500 \mathrm{~V}_{\mathrm{pp}}$. The collision cell RF was set to $3000-4000 \mathrm{~V}_{\mathrm{pp}}$, depending on the $\mathrm{m} / \mathrm{z}$ of the analyte ions. When tuning for the highest $\mathrm{m} / \mathrm{z}$ species (GroEL), the optimum RF amplitudes were not achieved with the commercial hardware, therefore RF amplitudes were set to the maximum values. Additionally, the collision cell transfer time and prepulse storage times were increased to 120-140 $\mu$ s and 15-25 $\mu \mathrm{s}$, respectively. When performing TIMS of protein complexes, the TIMS funnel RF was set to the maximum value of $350 \mathrm{~V}_{\mathrm{pp}}$. Finally, the collision cell gas flow was set to $85-100 \%$. TIMS was calibrated using the reduced mobilities from low concentration ESI tuning mix from Agilent (Santa Clara, CA). 


\section{Results and Discussion}

Several model proteins and protein complexes were chosen to evaluate the performance of the Bruker timsTOF Pro for transmission of high molecular weight species, efficient trapping within the ion mobility analyzer, and efficient dissociation of protein complexes. Trapped ion mobility spectrometry (TIMS) was performed for cytochrome c, avidin, streptavidin, cholera toxin B, and carbonic anhydrase. Protein complexes pyruvate kinase and GroEL were beyond the mobility range of the commercial instrument, however, their full mass spectra are shown below to showcase the transmission of high mass complexes and efficiency of in-source activation for improving spectral appearance and enhancing the ability to more confidently identify peaks convolved heavy salt adduction, often present in the analysis of biological analytes.

\section{Preservation of Native-like Protein Structures}

In order to preserve a protein close to its native structure in a TIMS measurement, it is imperative to minimize the heating of the protein in the ion source, throughout the TIMS analyzer, and in post-TIMS ion optics. Reducing the thermal heating and unintended ion activation during ESI can be achieved by employing a low dry gas temperature and a reduced electrospray voltage. Retention of even weaklybound peptide clusters in post-TIMS ion optics can be accomplished by reducing DC electric fields as described. ${ }^{21}$ Minimizing vibrational ion heating in the TIMS analyzer can be accomplished by minimizing translational-vibrational energy uptake due to (1) the axial DC electric field; (2) the radial RF electric field; and (3) space-charge effects and ion-ion interactions. ${ }^{22,}{ }^{23}$ In our experience, the most critical aspect is to minimize all DC electric fields in the regions prior to the mobility separation in TIMS. Hence, we apply a low DC bias between the deflector plate and entrance funnel $(\Delta 3)$, across the entrance funnel $(\Delta 4)$, and between the accumulation and mobility separation regions in the analyzer $(\Delta 6)$. Radial confinement of elevated ion densities in TIMS can increase the ion translational energy due to long range ion-ion interactions and power absorption from the RF electric field. At the same time, sufficient radial confinement of ions via the RF electric field is often required to ensure ion transmission through the TIMS analyzer. Hence, as a compromise between minimizing ion heating and optimizing ion transmission, we typically reduce the ion density by using low accumulation times and apply low-to-moderate RF amplitudes.

The specific settings that will maintain the structure of a given protein close to its native structure must generally be optimized for the system of interest. To provide a set of "soft" TIMS settings that can be used as a starting point for others in their own tuning, we discuss the retention of the $12.4 \mathrm{kDa}$ protein cytochrome c close to its native structure (Figure 1). To minimize thermal activation during ESI, we employ a dry gas temperature of $\sim 30^{\circ} \mathrm{C}$. We minimize collisional activation prior to the TIMS analysis by setting the DC voltage bias between the deflector and entrance funnel $(\Delta 3)$, across the entrance funnel $(\Delta 4)$, and between the accumulation and mobility separation regions in the analyzer $(\Delta 6)$ to $\Delta 3=20 \mathrm{~V}, \Delta 4=10 \mathrm{~V}$, and $\Delta 6=5 \mathrm{~V}$, respectively. We use an RF peak-to-peak amplitude of $300 \mathrm{~V}$ in the analyzer with an accumulation time of $70 \mathrm{~ms}$. We stress, however, that the values for these experimental settings, especially the RF amplitude and the accumulation times, are not universally valid and should not be applied to other protein samples without tuning. Instead, to produce a "soft" spectrum for a specific protein system, the operator is generally required to optimize their settings by following the principles of reducing ion heating described above and elsewhere. ${ }^{22,23}$ 

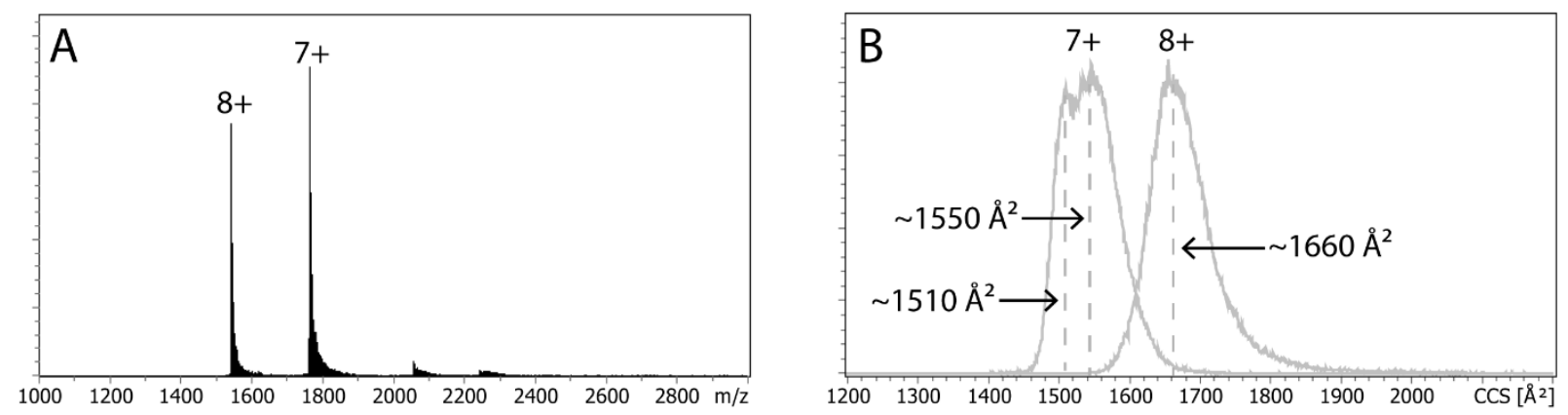

Figure 1. Full mass spectra (A) and extracted mobility distributions (B) for $30 \mu \mathrm{M}$ cytochrome $\mathrm{c}$ in $10 \mathrm{mM}$ aqueous ammonium acetate solution.

Figure 1A shows the mass spectrum obtained for cytochrome $c$. In line with a previous report from McLean et $\mathrm{al}^{16}$, charge states $7+$ and $8+$ dominate the spectrum. The ion mobility spectrum of charge state $7+$ shows a single peak centered at $\sim 1550 \AA^{2}$ with a shoulder at approximately $1510 \AA^{2}$ (Figure 1B). These values are consistent with cross sections of $\sim 1550 \AA^{2}$ and $\sim 1590 \AA^{2}$ observed by drift tube IMS ${ }^{16,17}$ and calculated for the $x$-ray crystal structure $\left(\sim 1565 \AA^{2}\right) .{ }^{28}$ The ion mobility spectrum recorded for charge state $8+$ displays a single feature centered at $\sim 1660 \AA^{2}$, also in line with the cross section expected for the x-ray structure. We stress that, in contrast to prior reports using a drift tube ${ }^{16}$ and TIMS $^{35}$, peaks with cross sections in the range of $1800 \AA^{2}$ to $2300 \AA^{2}$ corresponding to unfolded cytochrome c structures are not present in Figure 1B. This finding indicates that TIMS is able to produce "softer" spectra than drift tubes when appropriate "soft" settings are employed. Overall, the data shown here demonstrate the ability of the Bruker TIMS-Q-TOF platform to preserve native-like protein structures.

\section{Transmission and IMS of Protein Complexes}

Protein complexes avidin, streptavidin, and CTB were used to evaluate the spectral and IMS quality of the Bruker TIMS-Q-TOF mass spectrometer (Figure 2). Mobility distributions were extracted for individual charge states. 


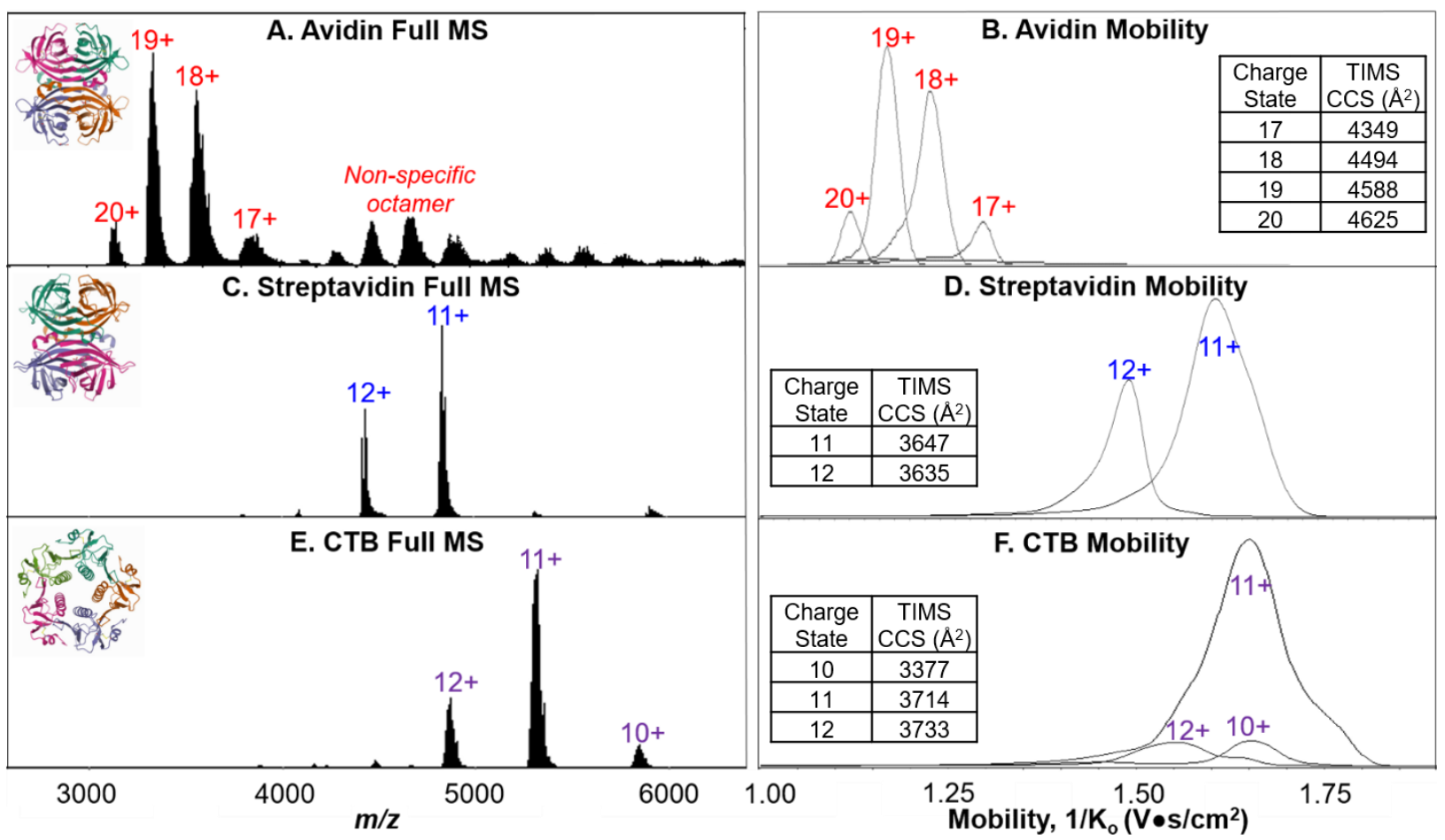

Figure 2. Full mass spectra and extracted mobility distributions and experimental CCS values for $20 \mu \mathrm{M}$ avidin (A, B, PDB 1AVE) in $200 \mathrm{mM}$ ammonium acetate, $5 \mu \mathrm{M}$ streptavidin (C, D, PDB 1SWB) in $160 \mathrm{mM}$ ammonium acetate and $40 \mathrm{mM}$ TEAA, and $5 \mu \mathrm{M}$ CTB (E, F, PDB: 1FGB) in $160 \mathrm{mM}$ ammonium acetate and 40 mM TEAA.

Avidin, a $58 \mathrm{kDa}$ homotetramer, was prepared in $200 \mathrm{mM}$ ammonium acetate at a final protein concentration of $20 \mu \mathrm{M}$. While this concentration is higher than those for the other protein complexes, we have included it here to showcase the transmission of non-specific oligomers at a higher mass to charge range (both octamer and 12-mer were observed (Figure 2A). When performing TIMS, the charge states of avidin were both well-defined and resolved (Figure 2B). It should be noted that avidin is a heavily glycosylated protein complex and hence the mass spectrum contains wider peaks when compared to streptavidin and CTB. ${ }^{36,37}$ Specifically, the wider distributions in $\mathrm{m} / \mathrm{z}$ of the $18+$ and $17+$ charge states are reflected by stronger fronting on the extracted mobility distributions.

Many nMS experiments involve the addition of a charge reducing agent, as lower charged precursors tend to have CCS distributions more closely mirroring known crystal structures and also yield more compact, native-like product ions upon activation. ${ }^{38}$ Therefore, streptavidin and CTB were both analyzed under charge-reducing conditions (Figure 2C and 2). For streptavidin, a $53 \mathrm{kDa}$ homotetramer, the corresponding mass spectra yield the expected native-like charge states and well-resolved peaks and mobility distributions were observed. The mobility distributions for the streptavidin charge states (Figure 2D) are much wider than for avidin, despite being lower in mass and less subject to glycosylation. Therefore, we attribute the increased width in mobility distribution to reaching the upper limit of the 
TIMS device, and thus the efficiency of trapping higher $m / z$ species at this range. This is further confirmed with the results of CTB (Figure 2F), a $58 \mathrm{kDa}$ homopentamer, which also yielded wide mobility distributions for the charge-reduced species. As the upper limit for mobility is approached, an RF driver of lower frequency would be needed to analyze protein complexes beyond this region. The experimental CCS $_{\text {TIMS }}$ values agree with previously reported literature (except for the proceeding exception) for the three protein complexes, suggesting native like structures are maintained. ${ }^{39}$ The CCS for the 10+ CTB pentamer is lower than that previously reported in the literature $\left(3370 \AA^{2} \text { here, compared to } 3910 \AA^{2}\right)^{34}$, however, we attribute this to charge stripping of the $12+$ or $11+$ precursor to form the $10+$ pentamer after the mobility analyzer and before TOF mass analysis. ${ }^{39}$

\section{Collision-Induced Dissociation of Streptavidin}

The use of tandem mass spectrometry further enhances the amount of structural information that can be gained in nMS experiments. Collision-induced dissociation (CID) is a gas-phase activation technique in which ions of interest undergo many low energy collisions with a background gas. Because protein complexes are large in mass and therefore high in degrees of freedom, high CID energies are required to achieve dissociation, particularly for charge-reduced species. Here, we performed CID in the commercial collision cell to evaluate the efficiency of dissociation for charge-reduced streptavidin (Figure 3).

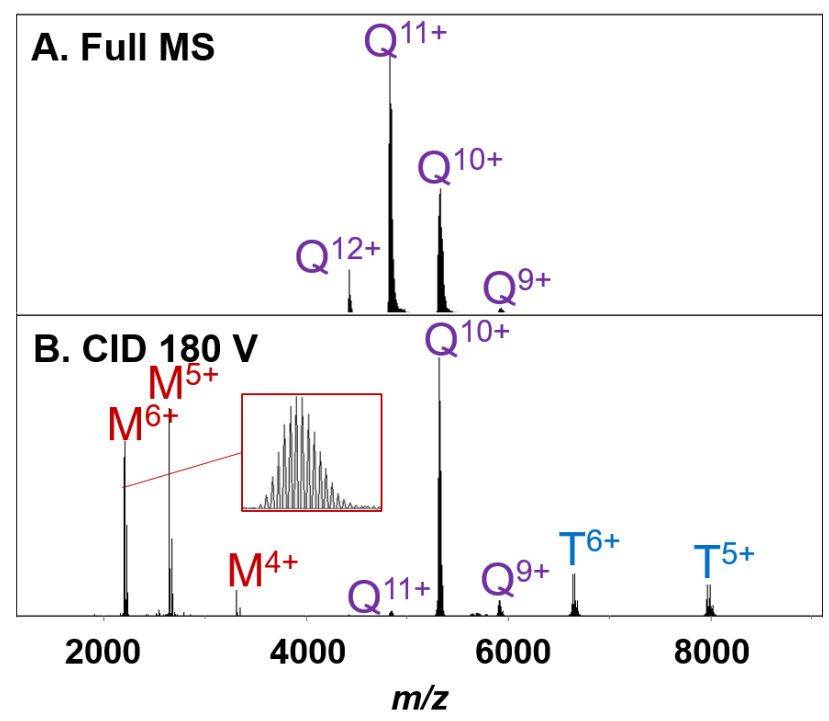

Figure 3. Streptavidin tetramer $(5 \mu \mathrm{M})$ in $160 \mathrm{mM}$ ammonium acetate with $40 \mathrm{mM}$ TEAA full MS (A). Collision cell CID $180 \mathrm{~V}$ of entire charge state distribution (B). 
Because we are beyond the upper limit of the analytical quadrupole's if driver, limited by the manufacturer to $\mathrm{m} / \mathrm{z} 3,000$, no mass selection was performed prior to activation. Collision cell CID of the entire streptavidin charge state distribution yielded the expected CID product ions. The tetramer fragmented to produce highly charged monomer and the correspondingly charged trimer. Dissociation was observed for CID energies as low as $150 \mathrm{~V}$ (out of a $200 \mathrm{~V}$ maximum).

\section{In-Source Activation of Pyruvate Kinase}

One challenge with analyzing large protein complexes using nMS is the presence of non-specific salt adducts, which broaden peaks in the resulting mass spectrum, resulting in poor apparent mass accuracy and poor apparent resolution (unresolved peaks). Therefore, in-source activation (isCID) is commonly used to knock off these adducts. The Bruker timsTOF Pro is equipped with in-source activation capabilities which are demonstrated below using pyruvate kinase in $200 \mathrm{mM}$ ammonium acetate (Figure 4). Note that is CID can cause restructuring of protein complexes and is recommended for MS but should be used with caution when native MS/MS is desired (e.g., SID, ExD, UVPD) for subunit connectivity studies or localization of ligands in a complex. 


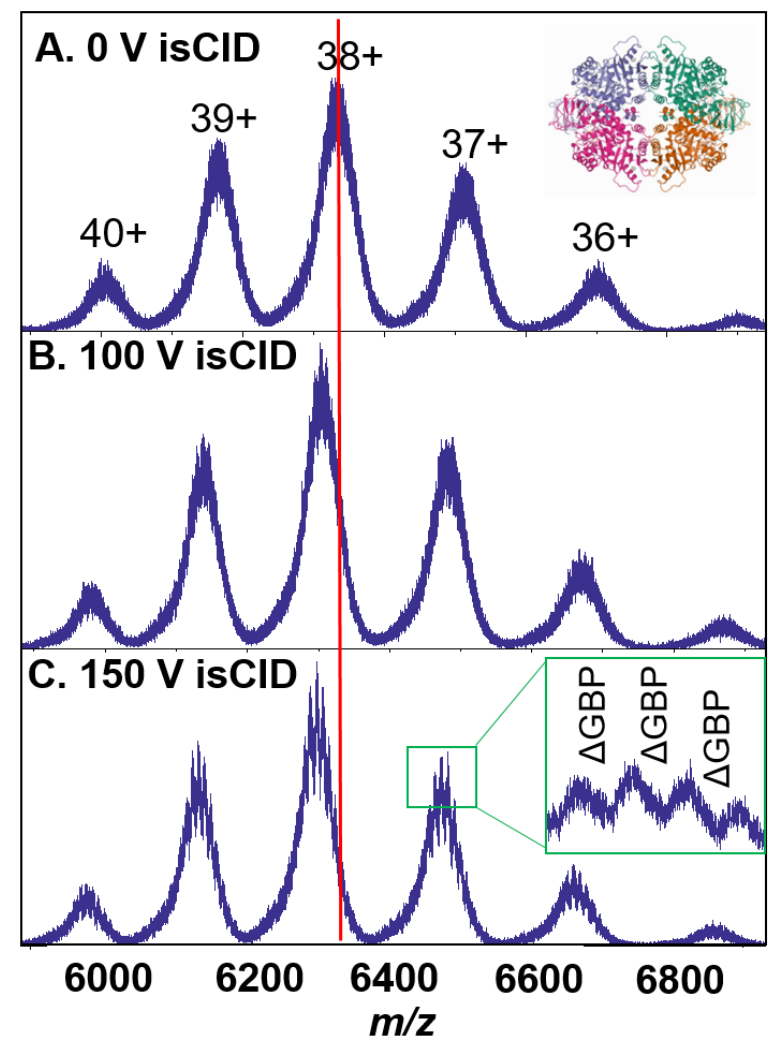

Figure 4. Pyruvate kinase $(5 \mu \mathrm{M})$ full MS with increasing amounts of in-source activation (isCID) to remove non-specific salt adducts. As salt is lost, the mass of the complex decreases, as shown by the red line placed at the centroid of the non-activated $38+$ charge state (A, B). At high isCID, GBP adducts start to be resolved $(\mathbf{C})$.

Pyruvate kinase is a $237 \mathrm{kDa}$ tetramer that forms adducts with the allosteric regulator 2,5-anhydro-Dglucitol, 1,6-bisphosphate (GBP, 324 Daltons), that is retained even with collisional activation. ${ }^{40}$ Without any in-source activation, the charge states of pyruvate kinase are wide, salt-adducted peaks (Figure 4A). However, as in-source activation is increased, the centroid of the charge states shifts to lower $\mathrm{m} / \mathrm{z}$, indicating the loss in mass from salt adducts (Figure 4B and C). At $150 \mathrm{~V}$ is CID, GBP additions begin to be resolved. These results suggest the in-source activation provided in the commercial timsTOF Pro is efficient at desalting large protein complexes.

\section{Transmission of a High Molecular Weight Protein Complex}

The upper $m / z$ for ion transmission was tested using GroEL, an $801 \mathrm{kDa} 14-m e r$. The full MS for GroEL is shown in Figure 5. The full mass spectrum of GroEL yields well resolved charge states, even at the highest $m / z$ region we analyzed. GroEL was able to be transferred throughout all ion optics in the instrument using all maximum RF $V_{p p}$ and low RF frequency settings available. However, GroEL was outside the commercial range of the TIMS device, as was pyruvate kinase, and would require hardware modifications to this region for the trapping of the large complex. 


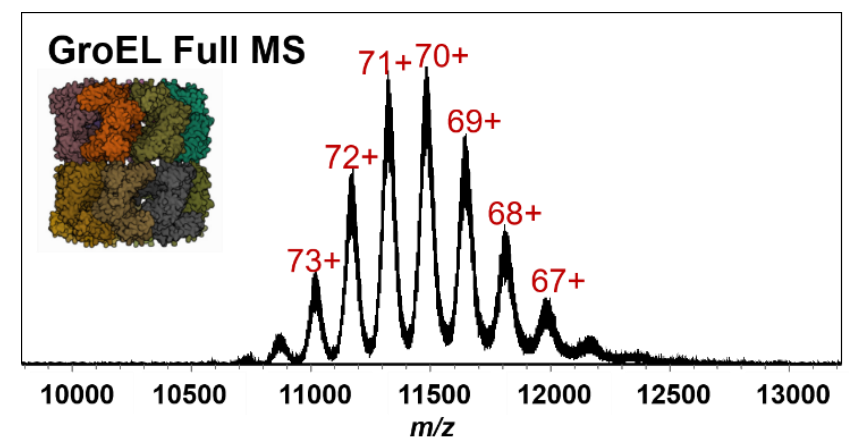

Figure 5. Full MS of GroEL (5 $\mu \mathrm{M})$ in $200 \mathrm{mM}$ ammonium acetate.

\section{TIMS of Carbonic Anhydrase and Ligand}

Proteins and protein complexes often rely on the binding of ligands to carry out their cellular functions. Many proteins undergo structural changes upon ligand binding, and therefore nMS and IMS are valuable analytical techniques to probe these differences. Protein-ligand complexes can be sensitive to mass spectrometer tuning, that is, ligand loss can occur with harsh, activating conditions. Here, the binding of inhibitor 1,3-benzenedisulfonamide (BS) to carbonic anhydrase was preserved through the TIMS analyzer (Figure 6).
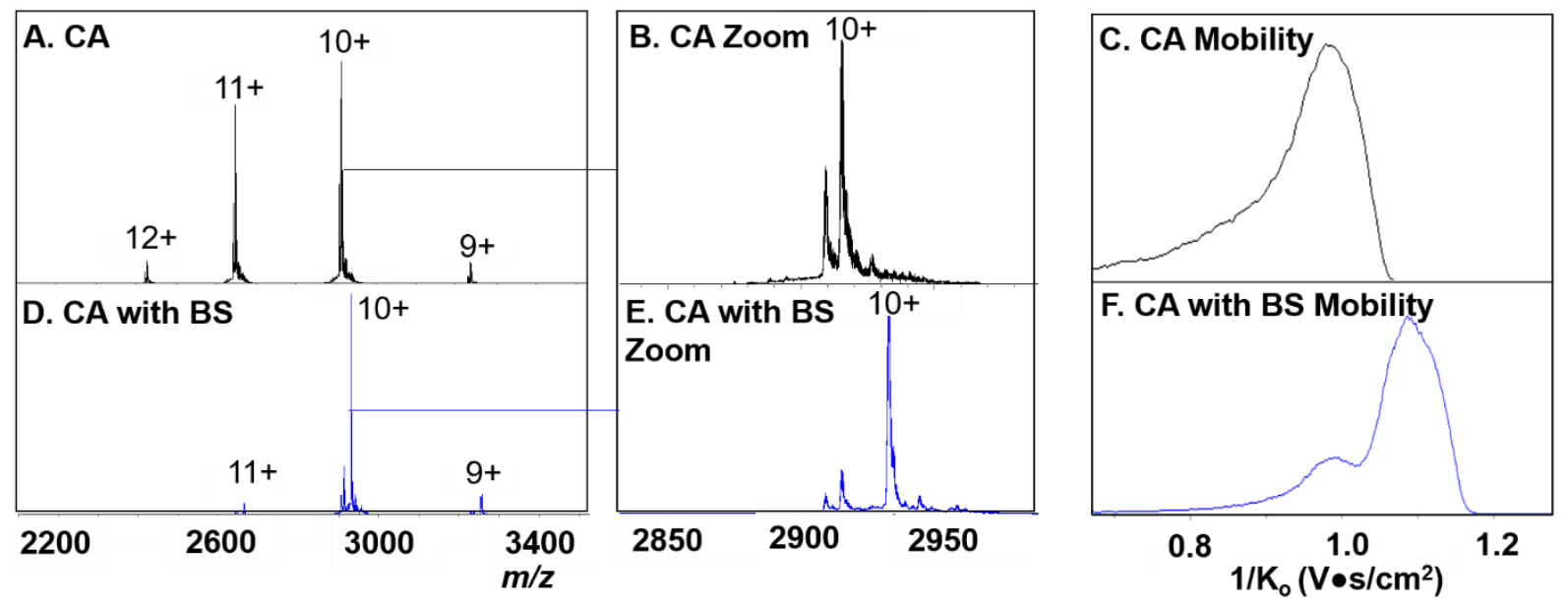

Figure 6. Carbonic anhydrase full MS and mobility for the $10+$ charge state (A, B, and C) compared to carbonic anhydrase with added ligand BS (D, E, and F).

Carbonic anhydrase is a monomeric protein $(30 \mathrm{kDa})$ that has well defined mechanistic activity, including the effect of inhibitor binding. ${ }^{41}$ A comparison of the full mass spectra and zoom-in of the $10+$ charge states with and without ligand added are shown (Figure 6A, B, D, and E). Binding of the ligand is retained throughout the entirety of the instrument, confirming the Bruker IM-Q-TOF MS can be used to investigate protein-ligand interactions. BS was only lost upon performing intentional CID in the collision cell. A shift 
toward lower mobility (higher $1 / K_{0}$ ) in the TIMS distribution was also observed upon ligand binding (Figure 6C and F), likely attributed to inhibitor binding rendering the conformationally mobile active site of carbonic anhydrase inactive, thus reducing catalytic activity of the enzyme. ${ }^{41,42}$ Further investigation, including molecular modeling and catalytic activity measurements will be required to provide specific insight into the binding effects on conformational shifts, however, detectable differences in gas-phase structures illustrates the application of ion mobility spectrometry in structural biology.

\section{Conclusions}

In this work we evaluated the use of a commercially available timsTOF Pro for native mass spectrometry applications. Multiple protein complexes, including avidin, streptavidin and cholera toxin B were analyzed for both full MS spectral quality and TIMS mobility distributions. Charge-reduced streptavidin and CTB began to reach the upper trapping limit of the TIMS device. However, CID performed in the collision cell was able to dissociate the large protein complexes, allowing for MS/MS experiments on native samples. Larger protein complexes pyruvate kinase and GroEL were outside the TIMS range of the commercial instrument, however, the complexes were still transferred throughout the instrument optics and detected, even demonstrating the applicability of in-source activation for sample clean up. Finally, the interaction between carbonic anhydrase and a ligand were maintained throughout TIMS analysis, demonstrating that the timsTOF Pro can be tuned to gently transfer samples without ligand loss. The Bruker timsTOF Pro, without complicated modifications, can be used to analyze native-like proteins and protein complexes without instrument modifications, essentially serving as a dual "omics" and native mass spectrometer to provide complementary structural biology information. Here we show the use of both an in-house nanoESI source and the commercially available ESI source for the analysis of native-like proteins. Instrument modifications, such as lower frequency RF drivers in both the TIMS and quadrupole may help to extend the range of the platform to larger protein complexes (greater than a few tens of kDa).

\section{Acknowledgements}

The authors would like to thank The Ohio State University Campus Chemical Instrument Center, Mass Spectrometry and Proteomics for allowing us to use their Bruker timsTOF Pro mass spectrometer (1S100D026945-01) and Dr. Sophie Harvey for help with initial experiments. We are also grateful to Yue (Linny) Ju and Guillaume Tremintin from Bruker Daltonics for providing insight on using the timsTOF Pro. This work was supported by the NIH P41 Resource for Native Mass Spectrometry Guided Structural Biology, P41GM128577. Support from the National Institutes of Health under grant R01GM135682 is acknowledged. 


\section{Author Information}

The authors declare the following competing financial interest: Mark E. Ridgeway and Melvin A. Park are employees of Bruker, which manufactures and sells the Bruker timsTOF Pro evaluated in this work.

\section{References}

1. Ward, A.B.; Sali, A.; Wilson, I.A.; Integrative Structural Biology. Science. 2013, 339, 913-915.

2. Heck, A.J.R. Native mass spectrometry: a bridge between interactomics and structural biology. Nat. Met. 2008, 5, 11, 927-933.

3. van den Heuvel, R.H.H. and Heck, A.J.R. Native protein mass spectrometry: from intact oligomers to functional machineries. Curr. Opin. Chem. Biol. 2004, 8, 5, 519-526.

4. Leney, A.C. and Heck, A.J.R. Native Mass Spectrometry: What is in the Name? J. Am. Soc. Mass Spectrom. 2016, 28, 5-13.

5. Fenn, J.B.; Mann, M.; Meng, C.K.; Wong, S.F.; Whitehouse, C.M. Electrospray ionization for mass spectrometry of large biomolecules. Science. 1989, 246, 4926, 64-71.

6. Katta, V. and Chait, B.T. Observation of the heme-globin complex in native myoglobin by electrospray-ionization mass spectrometry. J. Am. Chem. Soc. 1991, 113, 22, 8534-8535.

7. Ganem, B.; Li, Y.T.; Henion, J.D. Detection of noncovalent receptor-ligand complexes by mass spectrometry. J. Am. Chem. Soc. 1991, 113, 16, 6294-6296.

8. Laganowsky, A.; Reading, A.; Hopper, J.T.S.; Robinson, C.V. Mass spectrometry of intact membrane protein complexes. Nat. Protoc. 2013, 8, 4, 639-651.

9. Benesch, J.L.P.; Ruotolo, B.T.; Sobott, F.; Wildgoose, J.; Gilbert, A.; Bateman, R.; Robinson, C.V. Quadrupole-Time-of-Flight Mass Spectrometer Modified for Higher-Energy Dissociation Reduces Protein Assemblies to Peptide Fragments. Anal. Chem. 2009, 81, 1270-1274.

10. Chernushevich, I.V. and Thomson, B.A. Collisional Cooling of Large lons in Electrospray Mass Spectrometry. Anal. Chem. 2004, 76, 6, 1754-1760.

11. Sobott, F.; Hernández, H.; McCammon, M.G.; Tito, M.A.; Robinson, C.V. A tandem mass spectrometer for improved transmission and analysis of large macromolecular assemblies. Anal. Chem. 2002, 74, 6, 1402-1407.

12. Fort, K.L.; van de Waterbeemd, M.; Boll, D.; Reinhardt-Szyba, M.; Belov, M.E.; Sasaki, E.; Zschoche, R.; Hilvert, D.; Makarov, A.A.; Heck, A.J.R. Expanding the structural analysis capabilities on an Orbitrap-based mass spectrometer for large macromolecular complexes. Analyst. 2017, 143, 1, 100-105. 
13. van de Waterbeemd, M.; Fort, K.L.; Boll, D.; Reinhardt-Szyba, M.; Routh, A.; Makarov, A.A.; Heck, A.J.R. High-fidelity mass analysis unveils heterogeneity in intact ribosomal particles. Nat. Meth. 2017, 14, 3, 283-286.

14. Giles, K.; Pringle, S.D.; Worthington, K.R.; Little, D.; Wildgoose, J.L.; Bateman, R.H. Applications of a travelling wave-based radio-frequency-only stacked ring ion guide. Rap. Comm. Mass Spectrom. 2004, 18, 20, 2401-2414.

15. Campuzano, I.D.G. and Giles, K. Historical, current, and future developments of travelling wave ion mobility mass spectrometry: a personal perspective. Trends in Anal. Chem. 2019, 120, 115620.

16. May, J.C.; Jurneczko, E.; Stow, S.M.; Kratochvil, I.; Kalkhof, S.; McLean, J.A. Conformational Landscapes of Ubiquitin, Cytochrome C, and Myoglobin: Uniform Field Ion Mobility Measurements in Helium and Nitrogen Drift Gas. Int. J. Mass Spectrom. 2018, 427, 79-90.

17. Bush, M.F.; Hall, Z.; Giles, K.; Hoyes, J.; Robinson, C.V; Ruotolo, B.T. Collision Cross Sections of Proteins and Their Complexes: A Calibration Framework and Database for Gas-Phase Structural Biology. Anal. Chem. 2010, 82, 22, 9557-9565.

18. Meier, F.; Brunner, AD.; Koch, S.; Koch, H.; Lubeck, M.; Krause, M.; Goedecke, N.; Decker, J.; Kosinski, T.; Park, M.A.; Bache, N.; Hoerning, O.; Cox, J.; Räther, O.; Mann, M. Online Parallel Accumulation-Serial Fragmentation with a Novel Trapped Ion Mobility Mass Spectrometer. Mol. \& Cell. Prot. 2018, 17, 2534-2545.

19. Spraggins, J.M.; Djambazova, K.V.; Rivera, E.S.; Migas, L.G.; Neumann, E.K.; Fuetterer, A.; Goedecke, N.; Ly, A.; Van de Plas, R.; Caprioli, R.M. High-Performance Molecular Imaging with MALDI Trapped Ion Mobility Time-of-Flight (timsTOF) Mass Spectrometry. Anal. Chem. 2019, 91, 22, 14552-14560.

20. Steigenberger, B.; van den Toorn, H.; Bijl, E.; Greisch, J.; Räther, O.; Lubeck, M.; Pieters, R.J.; Heck, A.J.R.; Scheltema, R.A. Benefits of Collision Cross Section Assisted Precursor Selection (capsPASEF) for Cross-linking Mass Spectrometry. Mol. \& Cell. Prot. 2020, mcp.RA120.002094.

21. Kirk, S.R.; Liu, F.C.; Cropley, T.C.; Carlock, H.R.; Bleiholder, C. On the Preservation of Non-covalent Peptide Assemblies in a Tandem-Trapped Ion Mobility Spectrometer-Mass Spectrometer (TIMSTIMS-MS). J. Am. Soc. Mass Spectrom. 2019, 30, 7, 1204-1212.

22. Liu, F.C.; Kirk, S.R.; Bleiholder, C. On the Structural Denaturation of Biological Analytes in Trapped Ion Mobility Spectrometry- Mass Spectrometry. Analyst, 2016, 141, 3722-3730.

23. Bleiholder, C.; Liu, F.C.; Chai, M. Comment on Effective Temperature and Structural Rearrangement in Trapped Ion Mobility Spectrometry. Anal. Chem. 2020, 92, 24, 16329-16333. 
24. Hebert, A.S.; Prasad, S.; Belford, M.W.; Bailey, D.J.; McAlister, G.C.; Abbatiello, S.E.; Hugert, R.; Woulters, E.R.; Dunyach, J.; Brademan, D.R.; Westphall, M.S.; Coon, J.J. Comprehensive SingleShot Proteomics with FAIMS on Hybrid Orbitrap Mass Spectrometer. Anal. Chem. 2018, 90, 15, 9529-9537.

25. Bekker-Jensen, D.B.; Martinez-Val, A.; Steigerwald, S.; Rüther, P.; Fort, K.L.; Arrey, T.N.; Harder, A.; Makarov, A.; Olsen, J.V. A Compact Quadrupole-Orbitrap Mass Spectrometer with FAIMS Interface Improves Proteome Coverage in Short LC Gradients. Mol. Cell Proteomics. 2020, 19, 4, 716-729.

26. Bleiholder, C.; Liu, F.C.; Chai, M. Chapter: Calculation of Momentum Transfer Cross-Sections in lon Mobility-Mass Spectrometry: Fundamentals and Applications, New Developments in Mass Spectrometry. Royal Society of Chemistry, 2021.

27. Larson, E.J.; Roberts, D.S.; Melby, J.A.; Buck, K.M.; Zhu, Y.; Zhou, S.; Han, L.; Zhang, Q.; Ge, Y. HighThroughput Multi-attribute Analysis of Antibody-Drug Conjugates Enabled by Trapped Ion Mobility Spectrometry and Top-Down Mass Spectrometry. Anal. Chem., 2021, 93, 29, 1001310021.

28. Li, H.; Nguyen, H.H.; Ogorzalek Loo, R.R.; Campuzano, I.D.G.; Loo, J.A. An Integrated Native Mass Spectrometry and Top-Down Proteomics Method that Connects Sequence to Structure and Function of Macromolecular Complexes. Nat. Chem. 2018, 10, 2, 139-148.

29. Gault, J.; Liko, I.; Landreh, M.; Shutin, D.; Bolla, J.R.; Jeffries, D.; Agasid, M.; Yen, H.; Ladds, M.J.G.; Lane, D.P.; Khalid, S.; Mullen, C.; Remes, P.; Huguet, R.; McAlister, G.; Goodwin, M.; Vinter, R.; Syka, J.; Robinson, C.V. Combining Native and 'omics' Mass Spectrometry to Identify Endogenous Ligands Bound to Membrane Proteins. Nat. Methods. 2020, 17, 5, 505-508.

30. Zhou, M.; Jones, C.M.; Wysocki, V.H. Dissecting the Large Noncovalent Protein Complex GroEL with Surface-Induced Dissociation and Ion Mobility-Mass Spectrometry. Anal. Chem. 2013, 85, 8262-8267.

31. Yan, J.; Zhou, M.; Gilbert, J.D.; Wolff, J.; Somogyi, A.; Pedder, R. E.; Quintyn, R.S.; Morrison, L.J.; Easterling, M.L.; Pasa-Tolic, L.; Wysocki, V.H. Surface-Induced Dissociation of Protein Complexes in a Hybrid Fourier Transform lon Cyclotron Resonance Mass Spectrometer. Anal. Chem. 2017, 89, 895-901.

32. Snyder, D.T.; Panczyk, E.; Stiving, A.Q.; Gilbert, J.D.; Somogyi, A.; Kaplan, D.; Wysocki, V. Design and Performance of a Second-Generation Surface-Induced Dissociation Cell for Fourier Transform Ion Cyclotron Resonance Mass Spectrometry of Native Protein Complexes. Anal. Chem. 2019, 91, 14049-14057.

33. Snyder, D.T.; Panczyk, E.M.; Somogyi, A.; Kaplan, D.A.; Wysocki, V. Simple and Minimally Invasive SID Devices for Native Mass Spectrometry. Anal. Chem. 2020, 92, 11195-11203. 
34. Panczyk, E.M.; Snyder, D.T.; Ridgeway, M.E.; Somogyi, A.; Park, M.A.; Wysocki, V.H. Surfaceinduced Dissociation of Protein Complexes Selected by Trapped lon Mobility Spectrometry. Anal. Chem. 2021, 93, 13, 5513-5520.

35. Morsa, D.; Hanozin, E.; Gabelica, V.; De Pauw, E. Response to Comment on Effective Temperature and Structural Rearrangement in Trapped Ion Mobility Spectrometry. Anal. Chem. 2020, 92, 24, 16334-16337.

36. Bruch, R.C. and White III, H.B. Computational and structural heterogeneity of avidin glycopeptides. Biochemistry. 1982, 21, 21, 5334-5341.

37. Liu, F.C.; Cropley, T.C.; Ridgeway, M.E.; Park, M.A.; Bleiholder, C. Structural Analysis of the Glycoprotein Complex Avidin by Tandem-Trapped Ion Mobility Spectrometry-Mass Spectrometry (Tandem-TIMS/MS). Anal. Chem. 2020, 92, 6, 4459-4467.

38. Mehmood, S.; Marcoux, J.; Hopper, J.T.S.; Allison, T.M.; Liko, I.; Borysik, A.J.; Robinson, C.V. Charge Reduction Stabilizes Intact Membrane Protein Complexes for Mass Spectrometry. J. Am. Chem. Soc. 2014, 136, 49, 17010-17012.

39. Stiving, A.Q.; Jones, B.J.; Ujma, J.; Giles, K.; Wysocki, V.H. Collision Cross Sections of ChargeReduced Proteins and Protein Complexes: A Database for Collision Cross Section Calibration. Anal. Chem. 2020, 92, 6, 4475-4483.

40. Belov, M.E.; Damoc, E.; Denisov, E.; Compton, P.D.; Horning, S.; Makarov, A.A.; Kelleher, N.L. From Protein Complexes to Subunit Backbone Fragments: A Multi-stage Approach to Native Mass Spectrometry. Anal. Chem. 2013, 85, 23, 11163-11173.

41. Krishnamurthy, V.M.; Kaufman, G.K.; Urbach, A.R.; Gitlin, I.; Gudiksen, K.L.; Weibel, D.B.; Whitesides, G.M. Carbonic Anhydrase as a Model for Biophysical and Physical-Organic Studies of Proteins and Protein-Ligand Binding. Chem. Rev. 2008, 108, 3, 946-1051.

42. Glöckner, S.; Ngo, K.; Wagner, B.; Heine, A.; Klebe, G. The Influence of Varying Fluorination Patterns on the Thermodynamics and Kinetics of Benzenesulfonamide Binding to Human Carbonic Anhydrase II. Biomolecules. 2020, 10, 4, 509. 